East African Medical Journal Vol. 86 (Supplement) January 2009

STATUS OF HEALTH SECTOR STRATEGIC PLANS IN FIVE COUNTRIES OF THE WHO AFRICAN REGION

S. P. Barry, MD, MPH, Programme Manager, Health Policies and Service Delivery, L.G. Sambo, MD, MPH, Regional Director, S. Bakeera, MD, Masters in Health Management, Planning and Policy, Freelance Consultant, P.O. Box 2762, Kampala, Uganda, J. M. Kirigia PhD., Programme Manager, Health Financing and Social Protection, A.J. Diarra-Nama, PhD., Director, Health Systems and Services Development, WHO Regional Office for Africa, BP 06, Brazzaville, Congo

Request for reprints to: Dr. S. P. Barry, Health Policies and Services Delivery Programme, Division of Health Systems and Services Development, WHO Regional Office for Africa, BP 06, Brazzaville, Congo

\title{
STATUS OF HEALTH SECTOR STRATEGIC PLANS IN FIVE COUNTRIES OF THE WHO AFRICAN REGION
}

\author{
S. P. BARRY, L.G. SAMBO, S. BAKEERA, J. M. KIRIGIA and A.J. DIARRA -NAMA
}

\begin{abstract}
Objectives: To assess the adequacy of the existing strategic plans and compare the format and content of health sector strategic plans with the guidelines in selected countries of the African region.

Data source: The health strategic plans for Gambia, Liberia, Malawi, Tanzania and Uganda, which are kept at the WHO/AFRO, were reviewed.

Data extraction: All health strategic plans among the Anglophone countries (Gambia, Ghana, Kenya, Liberia, Malawi, Mauritius, Tanzania, Uganda, Zambia and Zimbabwe) that were developed after the year $\mathbf{2 0 0 0}$ were eligible for inclusion. Fifty percent of these countries that fitted this criterion were randomly selected. They included Gambia, Liberia, Malawi, Tanzania and Uganda.

The analysis framework used in the review included situation analysis; an assessment of appropriateness of strategies that are selected; well developed indicators for each strategy; the match between the service and outcomes targets with available resources; and existence of a clear framework for partnership engagement for implementation.

Data synthesis: Most of the strategic plans identify key ill health conditions and their contributing factors. Health service and resource gaps are described but not quantified in the Botswana, Gambia, Malawi, Tanzania strategic documents. Most of the plans selected strategies that related to the situational analysis. Generally, countries' plans had clear indicators. Matching service and outcome targets to available resources was the least addressed area in majority of the plans. Most of the strategic plans identified stakeholders and acknowledged their participation in the implementation, providing different levels of comprehensiveness.

Conclusion: Some of the areas that are well addressed according to the analysis framework included: addressing the strategic concerns of the health policies; identifying key partners for implementation; and selection of appropriate strategies. The following areas needed more emphasis: quantification of health system gaps; setting targets that are cognisant of the local resource base; and being more explicit in what stakeholders' roles are during the implementation period.
\end{abstract}

\section{INTRODUCTION}

Planning is essential for health sector development, and good plans are an essential component of health systems improvement (1). Whereas the presence of documented plans does not automatically result in improved health sector performance (1), a good health sector strategic plan does provide an entry point for support from collaborators and partners using a common framework for resource allocation 
and implementation. Such an arrangement creates an environment that is conducive for efficient resource use.

As part of its mandate to support quality of health planning in the region, the World Health Organization Regional Office for Africa (WHO/ AFRO) distributed in 2005 guidelines for developing national health policies and plans (10) whose aim is to provide policy makers, health planners, health systems managers and the main actors in public and private health sectors, useful orientations for the elaboration of national health policies and health strategic plans. This review assesses status of the strategic plans in five countries in light of the WHO guidelines.

\section{MATERIALS AND METHODS}

Sampling: All health strategic plans among the ten Anglophone countries that were developed after the year 2000 were eligible for inclusion. These countries are Gambia, Ghana, Kenya, Liberia, Malawi, Mauritius, Tanzania, Uganda, Zambia and Zimbabwe. Fifty percent of the countries that fitted this criterion were randomly selected. They include Gambia, Liberia, Malawi, Tanzania and Uganda.

Analysis framework:The five components of this analysis framework were extracted from the WHO guidelines for developing national health policies and plans developed by AFRO (2005). These components were also described by other experts in planning $(2,3)$ as part of a logical planning framework.

Situational analysis: Whereas the health policy provides the broad goals to improve performance of the sector, the health sector strategic plan lays out the implementation framework for how these broad goals will be achieved. It is therefore mandatory that the health sector strategic plan makes explicit reference to the policy. The content of the strategic plan are the thematic areas requiring intervention. It should therefore have a situational analysis that identifies key ill health conditions, contributing factors to pattern of health conditions, health service and resource gaps. Other aspects that need to be considered in the situational analysis are the context within which the strategic plan will be implemented. These contextual factors include - political will and commitment, parallel policies, financing levels, mechanisms and trends.

Does the plan select appropriate strategies based on the situational analysis: The strategic plan should present a clear framework for addressing identified areas of concern resulting into development of relevant strategies. The areas to be addressed include - health conditions and their distribution; health service and resource gaps; relevance of external environmental factors. The selection of each strategy needs to be justified in terms of effectiveness to address the identified concern. Also providing a baseline status for previous implementation of a selected strategy where applicable helps to justify the level of selected inputs.

Does the strategic plan have relevant and well-developed indicators for each strategy: Each strategic plan should have indicators that capture the multiple dimensions of the health sector objectives and priorities. Indicators should be stated in clear and precise language. The indicators should be valid and reliable. The time dimension and source of data for each indicator should be stated. This will give some indication as to the ease of data collection and analysis.

Does the plan match service and outcome targets to available resources: The purpose of matching service and outcome targets to available resources is to ensure that the plan is realistic and stands a chance of being implemented from the onset. This is assessed in two steps: (i) whether costs and funding sources are identified for all strategies; and (ii) whether resource gaps are explicitly identified for the expected service and outcome targets for each strategy.

The strategic plan should also share vision/ goals with other frameworks that financing and implementation partners subscribe to, e.g. Poverty Reduction Papers (PRSP), Millennium Development Goals (MDG), and Medium Term Expenditure 
Framework (MTEF). It should also be explicit in identifying areas that can be achieved by work of other sectors and partners in order to obtain consensus and commitment.

Does the strategic plan have a clear framework for appropriate partnership engagement during implementation: Assuming that the strategic plan has made explicit reference to the policy and that in turn policy development has been through a widely consultative process then one might say that the strategic plan has consensus on implementation direction and scope. However, it is important for the strategic plan to identify relevant partners, as well as their comparative advantages. There should be a clear conceptual framework for roles and mandate of each partner including opportunities and mechanisms for participation, and how they inter-relate.

\section{RESULTS}

The Gambia (4), Liberia (9) and Uganda (5) had both current policies and strategic plans. The current draft strategic plan (2007 - 2020) for Gambia has been developed concurrently with the health policy (2007 -2020). The Gambia plan addresses most of the strategic concerns of the health policy, mostly focusing on delivery of the minimum health package and supporting interventions. Notably, the strategic plan outlines activities to improve coordination of the multiple health sector partners. Although the policy has identified disparity in the demand and quality of services at different levels of health care, there is no explicit strategy that addresses this. Probably because the levels of disparity have not been quantified, the strategic plan tends to focus on universal improvement and access for all areas of service delivery. The National Health Plan of Liberia takes into consideration all the elements that are laid out in the updated national policy of 2006. The current strategic plan for Uganda is the second $(2005 / 06-2009 / 10)$ after the policy which was developed in 1999. Many of the strategic issues that were identified at the time are still relevant and are well expounded in the current strategic plan.

Most of the strategic plans identify key ill health conditions as well as the contributing factors to the pattern of ill health. Health service and resource gaps are described but not quantified in the Botswana, Gambia, Malawi, Tanzania (4, 6-8) strategic documents. The Botswana strategic plan acknowledges the need for cost reduction and an increase of the resource envelope but these too are not quantified. The Malawi strategic plan describes but does not quantify resource gaps; it does define an essential health package that can be financed with available resources (7). Tanzania describes gaps in the district and other health services but does not quantify them (8). A key constraint identified in the Gambia strategic plan is the ineffective management structure at the Department of State for Health (4). The Liberia strategic plan describes resource gaps and provides for quantification as part of it is implementation.

Some of the parallel policies that were identified included the introduction of sector wide approach (SWAP) reform in Botswana. The SWAP in Botswana was welcomed as an opportunity to streamline work-plan development and implementation (6). In Malawi, the risks of decentralisation were acknowledged and a cautious approach adopted by the sector (7). In Liberia, Uganda and Tanzania, the health sector was seen as critical to the achievement of the poverty reduction strategy and other local government reforms. Most of the policies mentioned in the Gambia policy were related to the health sector $(5,8-9)$.

Most of the plans select strategies that relate to concerns identified in the situational analysis. The strategic plans for all the five countries had relevant indicators. Matching service and outcome targets to available resources was the least addressed area of many of the strategic plans.

Most of the strategic documents identify who the stakeholders are for implementation of the plans, providing different levels of comprehensiveness. All strategic documents acknowledged the participation of a number of stakeholders.

\section{DISCUSSION}

Does the plan select appropriate strategies based on the situational analysis?

The Gambia strategic plan selected strategies that addressed key ill health conditions as well as contributing factors to ill health including poor environmental situation, unhealthy lifestyles, and low levels of health literacy. The Malawi and Liberia plans 
identified strategies that addressed the key ill health conditions and the limited resources by defining a basic package of services. The Tanzania strategic plan identifies strategies that improves health service delivery at the different levels of care (4, 7-9).

The baseline status for previous implementation of a strategy justifies the levels of selected inputs. Two of the strategic plans address this section quite comprehensively. The Malawi strategic plan provided a detailed analysis of the progress in the sector as well as the role of contextual factors in terms of strengths, weaknesses, opportunities, threats and key assumptions. It provides a baseline for many of the input, process and output indicators. The Uganda strategic plan provides the current performance as well as contextual setting for each of the strategies $(5,7)$. The three remaining strategic documents were less detailed, making it difficult to assess whether the selected level of inputs were justifiable for improving the baseline situation. The current draft of the Gambia strategic plan described progress for some but not all the strategies. Also, it does not quantify current performance for each strategy. The Tanzania strategic plan had a strong emphasis on how organisation and management at the different levels has influenced progress. However, the health system gaps that were identified at different organisational levels were not quantified $(4,8)$. The Liberia plan describes the baseline status and included plan for quantifying the identified gaps (9).

Did the strategic plan have relevant indicators and targets: The Gambia Strategic Plan had indicators that were relevant to the attainment of sector objectives but most of these were broad, which probably explained the absence of clear targets. Similarly, the Tanzania strategic plan identified indicators but not set performance targets for the implementation period $(4,8)$. The Liberia strategic plan had clear descriptions for attainment of the key objectives. It also sets indicators and targets for some of the key areas that were relevant to the attainment of the strategic objectives (9).

The Liberia, Malawi and Uganda documents selected appropriate indicators as well as set targets for the planned implementation period. In addition, the Uganda strategic plan provided a core set of indicators for monitoring progress agreed to by the different stakeholders. The intention to mostly use the existing health management information system in the Malawi and Uganda documents helped in ensuring the affordability and ease of data collection $(5,7)$.

Did the strategic plan identify costs and funding sources for all strategies: All the plans except that for Gambia identify costs and funding sources for the selected strategies in varying level of detail. The Malawi strategic plan provided detailed costs for each of the programme areas. This costing takes account forecasted resources and the absorptive capacity. The Tanzania strategic plan provided total resources projected to be available during the implementation of the plan but did not relate this to the strategic objectives. It was difficult to deduce how adequately selected strategies were financed. The Uganda strategic plan identified costs and funding sources for the strategies. It also made a provision for realigning the set targets with actual resources. The Liberia strategic plan costed each of the strategic objectives as well as identifying the source of funding (4, 7-9).

Did the strategic plan identify resource gaps for the expected service and outcome targets for each strategy: The Uganda and Malawi strategic plans mentioned the anticipated shortage in funding and that it will affect implementation of strategies and subsequently attainment of targets. Examining the anticipated shortage was critical for judging the likelihood of attaining set targets $(5,7)$. The Liberia strategic plan was cognisant of the funding deficits and included plans for a more comprehensive quantification at a later stage (9).

\section{Inclusiveness of process and identification of key partners for attainment of objectives}

The Tanzania and Uganda strategic plans identified main actors and outlined the implementation arrangements. Defining the implementation arrangements provided a basis for obtaining commitment for specific actions. For example, the Malawi strategic plan was said to have been jointly prepared with partners resulting in a memorandum of understanding for its implementation. The Liberia plan identifies partners that had a direct and synergistic contribution to attainment of its objectives.

The Gambia strategic plan identified some areas that could be implemented by other partners. However 
it did not identify who the main implementation partners were but made provision for this to be determined at a later date. Three of the plans -Malawi, Tanzania and Uganda share goals with the poverty reduction strategy papers - a framework that implementation partners were likely to subscribe to. This will facilitate joint implementation and resource pooling towards common objectives (4-9).

In conclusion, areas that were well addressed according to the analysis framework included: addressing the strategic concerns of the health plans (Gambia, Liberia and Uganda); identifying key partners for implementation (Malawi, Tanzania, and Uganda); and selection of appropriate strategies (all five countries).

Three areas that could benefit from more emphasis in the support process included: quantification of health system gaps; setting targets that are cognisant of the local resource base; and being more explicit in what stakeholders' roles were during the implementation period.

\section{COMPETING INTERESTS}

The authors declare that they had no competing interest.

\section{AUTHORS' CONTRIBUTIONS}

All the authors were involved in the conception, design, analysis and drafting of the manuscript.

\section{REFERENCES}

1. World Health Organization Regional Office for Africa: Health systems strengthening in the African region. Realities and opportunities. Brazzaville; 2007.

2. Green A. An introduction to health planning in developing countries. London: Oxford University Press; 1999.

3. Collins C. Management and organization of developing health systems. London: Oxford University Press; 1994.

4. Government of the Gambia: Health Master Plan 20072020. Banjul; 2007.

5. Government of the Republic of Uganda: Health Sector Strategic Plan II. 2005 / 06-2009/10. Kampala; 2005.

6. Government of the Republic of Botswana: Health policy and strategy framework. Gaborone; 2000.

7. Government of the Republic of Malawi: A joint programme of work for a health sector wide approach 2004-2010. Lilongwe; 2004.

8. The United Republic of Tanzania: Second Health Sector Strategic Plan. Dar es Salaam; 2008.

9. Government of Liberia: National Health Strategy for Liberia. Monrovia; 2007.

10. World Health Organization Regional Office for Africa: Guidelines for developing national health policies and plans. Brazzaville; 2005. 